\title{
Testing the efficacy of INtegral Cognitive REMediation (INCREM) in major depressive disorder: study protocol for a randomized clinical trial
}

Muriel Vicent-Gil ${ }^{1,3}$, Beatriz Raventós ${ }^{1}$, Eduardo D. Marín-Martínez', Sara González-Simarro ${ }^{1}$, Anabel Martínez-Arán², Caterina del Mar Bonnin², Joan Trujols', Josefina Pérez-Blanco ${ }^{1}$, Javier de Diego-Adeliño ${ }^{1}$, Dolors Puigdemont', Maria Serra-Blasco ${ }^{3}$, Narcís Cardoner ${ }^{3}$ and Maria J. Portella ${ }^{1 *}$ (D)

\begin{abstract}
Background: Given the limitation of pharmacological treatments to treat cognitive symptoms in patients with Major Depressive Disorder (MDD), cognitive remediation programs has been proposed as a possible procognitive intervention but findings are not conclusive. This study investigates the efficacy of an INtegral Cognitive REMediation (INCREM) that includes a combination of a Functional Remediation (FR) strategy plus a Computerized Cognitive Training (CCT) in order to improve not only cognitive performance but also the psychosocial functioning and the quality of life.
\end{abstract}

Methods: A single blind randomized controlled clinical trial in 81 patients with a diagnosis of MDD in clinical remission or in partial remission. Participants will be randomized to one of three conditions: INCREM (FR + CCT), Psychoeducation plus online games and Treatment As Usual (TAU). Intervention will consist in 12 group sessions, of approximately 110 min once a week. The primary outcome measure will be \% of change in psychosocial functioning after treatment measured by the Functional Assessment Short Test (FAST); additionally, number of sick leaves and daily activities will also be recorded as pragmatic outcomes.

Discussion: To our knowledge, this is the first randomized controlled clinical trial using a combination of two different approaches $(F R+C C T)$ to treat the present cognitive deficits and to promote their improvements into a better psychosocial functioning.

Trial registration: Clinical Trials NCT03624621. Date registered 10th of August 2018 and last updated 24th August 2018. Keywords: Cognitive remediation, Depression, Functional remediation, Computerized cognitive training, Clinical trial

\section{Background}

Cognitive dysfunction is considered a new treatment target to improve psychosocial functioning and enhance the quality of life in patients with Major Depressive Disorder (MDD) [1, 2]. Previous research suggests that cognitive symptoms explain the low rates of global

\footnotetext{
* Correspondence: mportella@santpau.cat

'Department of Psychiatry, Hospital de la Santa Creu i Sant Pau, Centro de Investigación Biomédica en Red de Salud Mental (CIBERSAM), Institut d'Investigació Biomèdica Sant Pau (IBB-Sant Pau), Universitat Autònoma de Barcelona (UAB), Sant Antoni Ma Claret 167, 08025 Barcelona, Catalonia, Spain

Full list of author information is available at the end of the article
}

recovery and functional disability [3-5]. In fact, current pharmacological treatments for MDD have not been considered useful for the improvement of cognitive dysfunction probably because they are focused on improving mood [6]. The only antidepressants that seem to have a potential procognitive impact are duloxetine and vortioxetine [7-9], but further studies are needed to clarify these findings.

Regarding non-pharmacological treatments, Cognitive Remediation (CR) is a psychotherapeutic approach that has shown improvement in cognition in other neuropsychiatric disorders $[10,11]$ However, only few studies

(c) The Author(s). 2019 Open Access This article is distributed under the terms of the Creative Commons Attribution 4.0 International License (http://creativecommons.org/licenses/by/4.0/), which permits unrestricted use, distribution, and 
have been conducted in MDD patients [12-15]. The design features of the interventions differ from one another, making it difficult to demonstrate their efficacy. Moreover, samples included in CR studies were very heterogeneous with different disease burden, which would have impeded conclusions about the specific effect of CR for MDD. Analyses were also limited by the fact that not all patients show the same profile of cognitive impairment. It is estimated that $50 \%$ of depressed patients [16, 17] present enduring cognitive deficits, which would significantly interfere in workplace and in psychosocial functioning, while another large percentage of patients do not suffer from cognitive dysfunction.

Apart from that, most of CR programs have been based on neurological models designed to reverse cognitive deficits acquired after or associated with neurological conditions $[18,19]$, while the focus of CR programs in psychiatric diseases should be more directed in improving everyday functioning rather than merely recovering cognitive losses. Computerized Cognitive Training (CCT), which is based on cognitive exercises and games, appears to be, by contrast, an optimal method to improve cognitive functioning in affective disorders due to the flexibility given to adjust the tasks to the needs of each patient. In addition, and according to a recent meta-analysis [20], CCT improved depressive symptomatology and everyday functioning in patients with MDD, though producing inconsistent effects on cognition.

The scarce evidence on the efficacy of cognitive interventions may have prevented a broad implementation of such programs in the clinical setting. Moreover, research should define how cognitive remediation programs are to be administered (e.g., number of sessions), what cognitive domains are to be trained, and how this intervention can impact on psychosocial functioning. Therefore, there is a strong need of randomized clinical trials so as to demonstrate the efficacy of such interventions $[14,15]$.

Recently, one new 'ecological' intervention named Functional Remediation (FR) has been designed with the aim of transferring cognitive improvements to the daily functioning, by using neurocognitive techniques and training but also through psychoeducation on cognition and problem-solving. The FR program includes modeling techniques, role-playing, verbal instructions, self-instructions, positive reinforcement and meta-cognitive cues using real-life problems [21]. FR in bipolar disorder has shown significant enhancement of functional outcomes as well as subsyndromal symptomatology [2224]. This is a group intervention and, thus, it is difficult to tailor the tasks to the specific needs of each individual.

Considering the above, the evidence accumulated indicates that the most adequate intervention to treat cognitive symptoms in MDD would be a combination of a group FR plus a personalized CCT. The inclusion of both aspects will allow the intervention to focus on the present deficits through the formation of new strategies with compensatory techniques and promoting their use into everyday life.

\section{Aims of the study}

This study aims to show the efficacy of an INtegral Cognitive REMediation (INCREM) program specificallydesigned for MDD patients so as to improve psychosocial functioning through the treatment of cognitive symptoms.

Primary research aims:

- To develop and to prove the efficacy of INCREM; this includes a FR program and a CCT.

- To improve psychosocial functioning in MDD patients.

- To decrease the rate of patients with enduring cognitive symptoms, and to reduce the risk of further relapses.

Secondary research aims:

- To establish cognitive profiles in MDD patients, to tailor the Integral Cognitive Remediation therapy so as to achieve full remission.

- To increase the effect size of the intervention by combining traditional group sessions plus computerized individual sessions.

- To study the specific elements (cognitive and psychosocial aspects) that mediate clinical and functional improvement, which can be ascertain through well-being and decrements in cognitive complaints.

\section{Methods and Design}

This is a single blind, randomized controlled clinical trial approved by the Research Ethics Board of the Hospital de la Santa Creu i Sant Pau. All participants will receive extended information about the study and must give their written informed consent prior to the inclusion. The study will be carried out in accordance with the ethical principles of the declaration of Helsinki and Good Clinical Practices, complying with data protection laws with the anonymization of all the information collected (Data Protection Act 2018).

\section{Participants}

Patients aged between 18 to 60 years with a diagnosis of MDD (DSM-5 criteria) will be recruited from the psychiatry department of the Hospital de la Santa Creu i Sant Pau in Barcelona (Catalonia, Spain) to participate in the present study. This center covers a population of 
400.000 inhabitants, and therefore, the recruitment will be achieved. All patients will have to be in clinical remission or in partial remission, defined by scores below 14 in the HDRS-17. To be allocated in one of the treatment arms, patients will have to display objective cognitive deficits (measured with the Screening for Cognitive Impairment in Psychiatry - SCIP, defined by a score below 80$)[25,26]$ together with psychosocial dysfunction, defined by a score from 12 to 20 (mildly impaired) in the FAST [27]. All patients will continue with their usual pharmacological treatment. Exclusion criteria are: i) an intelligence quotient (IQ) below 85 , ii) any medical condition that may affect neuropsychological performance, iii) presence of any comorbid psychiatric condition including non-nicotine substance use disorders on the previous three months, iv) having received electroconvulsive therapy on the previous year or psychological intervention in the previous 6 months.

\section{Study design}

Figure 1 provides a schedule of the study. Detailed oral and written information about the study will be provided to all potential candidates to participate in the study. If they are interested in participate, informed consent will be signed and demographic, clinical, neuropsychological and functional assessments will be performed by an experienced clinical neuropsychologist. After the fulfillment of the inclusion criteria, patients will be randomized to one of the three possible treatment options: INCREM program which includes both a FR program adapted to depression, and a CCT; Psychoeducation plus online mental skill games; and Treatment as Usual (TAU). A more detailed explanation of the two active treatment arms is provided below. An independent statistician from the Department of Epidemiology (Hospital de la Santa Creu i Sant Pau) will carry out the randomization by means of computer-generated random numbers. In order to ensure balanced sample sizes across groups over time, a block randomization method will be used. The block size will be set to 27 , so as to have 9 patients per treatment arm, in 3 consecutive blocks. Blocks will then be randomly chosen to determine patients' final assignment. Another experienced clinical researcher will assign participants to the corresponding intervention. The psychologists conducting treatment interventions will be different from the clinical neuropsychologist who will carry out the assessments and who will be blind to the treatment assignment. The intervention will be discontinued in case of request by the participant. Any other concomitant psychological treatment will not be permitted during the trial. Finally, to ensure adherence to intervention, after one unjustified absence rated by the psychologist, a phone call will be done prior to the next session.
After 3 months of treatment (12 sessions) efficacy measures will be collected: Functioning Assessment Short Test (FAST) [28], Perceived Deficit Questionnaire (PDQ-20) [29, 30], Hamilton Depression Rating Scale (HDRS-17) [31, 32], Remission from Depression Questionnaire (RDQ) [33] and 36-Item Short Form Health Survey, Version 2 (SF-36-V2) [34]. Then, 6 months after the intervention, a complete demographic, clinical, neuropsychological and functional assessment will be performed in order to evaluate the eventual long term effects. To promote retention and interest in the study by the participants, a detailed report about their evolution will be provided to them.

Estimation of sample size was set at 27 participants in each treatment arm, assuming a minimum difference on 6 points on the FAST (primary outcome), and considering a bilateral significance level of $5 \%$ and a statistical power of $80 \%$, adjusted by a $20 \%$ rate of dropouts.

\section{Demographic and clinical assessment}

A semi-structured interview will be used to assess demographic and clinical variables at baseline. Demographic assessment will include gender, age, years of education, age at which schooling was completed, work situation, work adjustment, marital status, cohabiting characteristics, and the number of children if any.

The Cognitive Reserve Assessment Scale in Health (CRASH; in press) will be included so as to estimate the cognitive reserve. It is a semi-structured interview-based scale that evaluates three domains considered fundamental in the construct of cognitive reserve: education, occupation and intellectual and leisure activities.

The clinical evaluation will cover age at illness onset, number of previous episodes, age of first psychiatric hospitalization, number of hospitalizations, period of clinical stability, seasonal pattern, presence of rapid cycling depression, presence of melancholy, atypical depression and psychotic symptomatology during depression, comorbidities in axis I, II and III, life history of suicidal ideation, life history of suicide attempts, number of attempts, method and medical severity used, and family history of psychiatric disorders and suicide. The HDRS-17 will be used to evaluate current depressive symptoms and assess clinical changes. In addition, the RDQ will be used to evaluate the patient-perceived remission that includes subscales for symptoms of depression, other symptoms such as anxiety and irritability, coping ability, positive mental health, functioning, life satisfaction and a general sense of well-being. These two scales will be analyzed as secondary clinical outcomes to evaluate the possible lasting effects. 


\begin{tabular}{|c|c|c|c|c|c|}
\hline & \multicolumn{5}{|c|}{ STUDY PERIOD } \\
\hline & Enrolment & Allocation & Intervention & $\begin{array}{c}\text { Post- } \\
\text { Intervention }\end{array}$ & End point \\
\hline TIMEPOINT & $-t 1$ & to & $t 1$ & t2 & t3 \\
\hline \multicolumn{6}{|l|}{ ENROLMENT: } \\
\hline \multirow{4}{*}{$\begin{array}{r}\text { Eligibility screen } \\
\text { Explanation of the study } \\
\text { Informed consent } \\
\text { Allocation }\end{array}$} & $\mathrm{x}$ & & & & \\
\hline & $x$ & & & & \\
\hline & $x$ & & & & \\
\hline & & $x$ & & & \\
\hline \multicolumn{6}{|l|}{ INTERVENTIONS*: } \\
\hline \multirow{2}{*}{$\begin{array}{l}\text { INCREM (FR plus CCT) } \\
\text { Psychoeducation plus online games }\end{array}$} & & & $x$ & & \\
\hline & & & $\mathrm{x}$ & & \\
\hline$T A U$ & & & $x$ & & \\
\hline \multicolumn{6}{|l|}{ ASSESSMENTS**: } \\
\hline \multirow{4}{*}{$\begin{array}{r}\text { Demographic Assessment } \\
\text { Clinical Assessment } \\
\text { Neuropsychological Assessment } \\
\text { Outcome measures }\end{array}$} & $x$ & & & & $x$ \\
\hline & $x$ & & & $x$ & $x$ \\
\hline & $\mathrm{x}$ & & & & $x$ \\
\hline & $\mathrm{x}$ & & & $\mathrm{x}$ & $\mathrm{x}$ \\
\hline \multicolumn{6}{|c|}{$\begin{array}{l}\text { Footnote: } \\
\text { INCREM: INtegral Cognitive REMediation; FR: Functio } \\
\text { TAU: Treatment As Usual. } \\
\text { *Interventions will consist in } 12 \text { sessions, once a week. } \\
\text { **Demographic, clinical, Neuropsychological assessmer } \\
\text { Methods and Design section. }\end{array}$} \\
\hline \multicolumn{6}{|c|}{ Fig. 1 Schedule of enrolment, interventions, and assessments } \\
\hline
\end{tabular}

\section{Neuropsychological assessment}

Following the clinical assessment, a neuropsychological battery of tests will be administered by experienced neuropsychologists at the beginning of the study and at 12 months after intervention. It comprises standardized tests to assess attention, working memory, verbal and visual memory, processing speed and executive function. Premorbid Intelligence will be estimated with Vocabulary and Block Design subtests of the Wechsler Adult Intelligence Scale version-IV (WAIS-IV) [35]. Attention and verbal working memory performance will be evaluated with Digit forward and backward (WAIS-IV). The Conners Continuous Performance Test 3rd Edition (CPT-III) [36] will be used to assess sustained attention. Verbal learning memory will be measured by the California Verbal Learning Test (CVLT) [37]. Copy and recall of the Rey-Osterrieth Complex Figure (ROCF) [38] will be used to evaluate visuoconstructive abilities and visual memory. Processing speed will be measured by the Digit Symbol Substitution Test (DSST; WAIS-IV) and the Trail Making Test Part A (TMT-A) [39]. Finally, executive functioning will be measured with the Trail Making Test Part B (TMT-B) [39], the Stroop Color and Word Test (STROOP) [40], the Wisconsin Card Sorting Test (WCST) [41], the Tower of London (TOL) [42] and the Category Fluency and PMR (adapted for Spanish speaking population) $[43,44]$. TMT-B is used to evaluate set-shifting abilities, STROOP interference is used to evaluate difficulties with response inhibition, WCST assess abstract reasoning ability and the ability to shift cognitive strategies in response to changing environmental contingencies, TOL is used to detect deficits in planning and problem-solving and Category Fluency and PMR are used to measure semantic and phonemic verbal fluency, respectively. 


\section{Intervention}

Selected patients are going to be randomized to three possible intervention arms: INCREM; PSYCHOEDUCATION + online games; and Treatment as Usual (TAU).

INCREM will consist of 12 sessions, of approximately 110 min once a week, involving a FR and a CCT. FR program was originally developed for bipolar patients [45] and it will be adapted for depressed patients creating a more intensive program focused on executive functions, memory and attention. The sessions aim at improving daily functioning based on ecological tasks where they need to use compensatory techniques. The specific explanation of the sessions is developed in Table 1. CCT will be applied right after FR group sessions in individual computer sessions during $20 \mathrm{~min}$. The modules of training will be facilitated by the licensed game-like software CogniFit (https://www. cognifit.com). This neurocognitive stimulation program consists of a battery of tasks that allows depressed patients to improve their cognitive skills depending on their individual performance throughout different activities.

PSYCHOEDUCATION will consist of 12 sessions, 90 min once a week involving psychoeducation sessions plus $20 \mathrm{~min}$ online non-directed game playing. This treatment arm has been designed as the control intervention arm due to its beneficial effects for depression. The material for the psychoeducation intervention has been specifically designed for the study and is based on validated treatment protocols [46-48]. Information about the disease is provided during the sessions so as to gain knowledge and strategies to cope with their own difficulties and to improve their quality of life. Therefore, the intervention will be divided in two modules: the first module (sessions 1 to 4 ) will consist in providing

Table 1 Content of the 12-week Functional Rehabilitation Program

\begin{tabular}{|c|c|}
\hline Session & Topics Covered \\
\hline 1. What is attention? (I) & Strategies of concentration \\
\hline 2. What is attention? (II) & Strategies to improve attention \\
\hline 3. Reading & Recovering the habit of reading \\
\hline 4. What is memory? (I) & Mnemonic strategies \\
\hline 5. What is memory? (II) & $\begin{array}{l}\text { The use of diaries and external } \\
\text { aids }\end{array}$ \\
\hline 6. What are executive functions? (I) & Self-instructions \\
\hline 7. What are executive functions? (II) & $\begin{array}{l}\text { Programing activities, prioritization } \\
\text { and time management }\end{array}$ \\
\hline 8. What are executive functions? (III) & Problem solving strategies \\
\hline 9. What are executive functions? (IV) & Working memory strategies \\
\hline 10. Stress & Stress and neurocognition \\
\hline 11. Communication & $\begin{array}{l}\text { Communication skills and } \\
\text { assertiveness }\end{array}$ \\
\hline 12. Autonomy & Independence and autonomy \\
\hline
\end{tabular}

information about the disease and its treatment, and the second one (sessions 5 to 12) will consist of introducing different cognitive-behavioral techniques that have proven to be effective for depression. The specific explanation of the sessions is developed in Table 2. The online game will be applied right after the psychoeducation sessions during $20 \mathrm{~min}$. The games will be facilitated by a free mini-game online website (www.frivplus.com). No records will be gathered on this last part, as it is only used to make comparable the two active treatment arms.

TAU will consist in giving the usual treatment to patients according to accepted standards for depression (except psychotherapy which will not be allowed 6 months before the study nor during it).

\section{Outcome measures}

The primary outcome measure of the study will be the change in psychosocial functioning after treatment, which will be measured by means of the FAST. The FAST is a scale created to assess disability in functioning in mental health which contains 24 items evaluating autonomy, occupational functioning, cognitive functioning, financial issues, interpersonal relationships and leisure time. The scores range from 0 to 72 , with higher scores indicating a greater disability. A sum of $\geq 12$ represents a mildly to severe functional impairment in patients with bipolar disorder [27]. Given the complexity of the possible effects of the intervention, changes in pragmatic variables of psychosocial functioning will also be evaluated: number of sick leaves and number of daily activities.

Table 2 Content of the 12-week Psychoeducational Program

\begin{tabular}{|c|c|}
\hline Session & Topics Covered \\
\hline 1. What is depression? (I) & Concept of depression \\
\hline 2. What is depression? (II) & $\begin{array}{l}\text { Definition, symptoms, prevalence } \\
\text { and course }\end{array}$ \\
\hline 3. Etiology of depression & $\begin{array}{l}\text { Biopsychosocial model, psychobiology } \\
\text { and psychological theories }\end{array}$ \\
\hline 4. Treatment of depression & $\begin{array}{l}\text { Pharmacological, psychological and } \\
\text { other treatment options }\end{array}$ \\
\hline 5. Behavioral Activation (I) & Introduction to Behavioral Activation \\
\hline 6. Behavioral Activation (II) & Exposition to positive activities \\
\hline 7. Cognitive Distortions & $\begin{array}{l}\text { Cognitive distortions, automatic } \\
\text { negative thoughts and cognitive } \\
\text { restructuring technique }\end{array}$ \\
\hline 8. Anxiety & Anxiety management strategies \\
\hline 9. Social Skills (I) & $\begin{array}{l}\text { Social skills deficits and } \\
\text { communicational styles }\end{array}$ \\
\hline 10. Social Skills (II) & Social skills training \\
\hline $\begin{array}{l}\text { 11. Problem-Solving } \\
\text { Technique }\end{array}$ & Problem-Solving training \\
\hline 12. Relapse Prevention & $\begin{array}{l}\text { Summary of the sessions and relapse } \\
\text { prevention }\end{array}$ \\
\hline
\end{tabular}


Different secondary outcome measures will be analyzed: i) the change in cognitive performance across cognitive domains (through composite scores of the different cognitive domains and a global composite cognitive score); ii) change in subjective appraisal of cognitive functioning evaluated with the PDQ-20 which is a self-reported questionnaire that measures the patients' perception of their cognitive functioning. It consists of 20 items assessing attention, retrospective memory, prospective memory, and planning and organization; iii) change in depressive symptoms with the HDRS-17); iv) change in patients' perspective of clinical remission with the RDQ; and v) change in quality of life assessed with the SF-36-V2. It evaluates the patients' health-related quality of life gauging eight sections: vitality, psychical functioning, bodily pain, general health perceptions, physical role functioning, emotional role functioning, social role functioning and mental health.

\section{Statistical analysis plan}

Data will be collected in three different moments: baseline, post-intervention and follow-up through a logbook data collection. Then, an online application called Clinapsis will be used for data collection, which ensures anonymisation of data (through encoding system). This system will grant public access to the full protocol and dataset at participant-level for further analysis beyond this proposal.

The data will be analyzed using the Statistical Package of Social Sciences (SPSS, IL, Chicago, version 20) and R Package (version 3.1.2). First, baseline differences between groups will be investigated using chi-square tests and ANOVAs depending on the nature of the variables. Secondly, mixed-models will be carried out to assess the impact of the three different treatment options on the psychosocial functioning scores assessed with the FAST scale (primary outcome measure). Effect sizes of interventions will be calculated. Secondary variables will also be analyzed with the same statistical approach. Mixed repeated measures ANOVAs will be carried out to investigate the long-term effects of interventions on secondary outcomes. Finally, in order to investigate predictors of treatment response, a study of mediators and moderators will be conducted. For the main statistical analysis, the Last Observation Carried Forward method will be used to minimize the effect of dropout rates at 6 months of follow-up after intervention.

\section{Discussion}

Cognitive impairment is nowadays considered a core symptom of MDD and a critical determinant of psychosocial and workplace outcomes, as well as health outcomes such as the health-related quality of life. CR has been used to treat these cognitive deficits suggesting significant improvements in cognition but with small effect sizes for psychosocial functioning. This study aims to improve psychosocial functioning through the improvement in cognitive performance. To our knowledge, this is the first randomized clinical trial using an INtegral Cognitive REMediation (INCREM) composed by 12 sessions of a FR program and a CCT.

The major strength of this study is the combination of two different approaches at the same time: a group format therapy together with a computerized program that allows a personalized delivery of the intervention. This synergistic approach may represent the path to optimize treatment response and ensure better functional outcomes in patients with MDD. The presence of an active control group beyond treatment as usual (TAU) is another relevant strength of the study. A large battery of primary and secondary outcome measures will be used to evaluate the efficacy of the intervention. $\mathrm{Pa}$ tients will be approached six months after finishing the intervention to assess the maintenance of possible changes. Furthermore, this study will take into account the specific cognitive deficits of each patient by adapting CCT to individual performance.

A potential limitation of the present study is inherent to any clinical trial, given that participation and follow-up of the interventions could be more complicated. A $20 \%$ of dropouts are calculated throughout the intervention and/or follow-up has been estimated, but this percentage could be higher, which would limit the power of the analysis. Patients should be in remission or in partial remission with cognitive deficits and difficulties in daily life, so that recruitment and inclusion might be more complicated. Concomitant medication might also represent a possible limitation because of side effects or even procognitive effects of newer drugs. Medication regimes will be recorded during the different phases of the study and then will be taken into account in the analyses. In any case, in the clinical practice, polymedicated patients are the rule rather than the exception. Hence, it might limit internal validity but it is strength for the generalization of the results.

\section{Conclusion}

The presence of residual cognitive symptoms, observed in the clinical settings, may impede the achievement of full remission in patients with depression. Therefore, effective cognitive treatments are an unmet need. If the results of this study are conclusive, the INCREM could be added to the therapeutic arsenal for depression. This non-pharmacological treatment may seem expensive as it consists in 12 sessions, but if one considers the direct and indirect social and healthcare costs of depression [49], then targeting cognitive symptoms with a cognitive program will likely be cost-effective. 


\section{Abbreviations}

CCT: Computerized Cognitive Training; CPT-III: Conners Continuous Performance Test 3rd Edition; CR: Cognitive Remediation; CRASH: Cognitive Reserve Assessment Scale in Health; CVLT: California Verbal Learning Test; DSST: Digit Symbol Substitution Test; FAST: Functioning Assessment Short Test; FR: Functional Remediation; HDRS-17: Hamilton Depression Rating Scale; INCREM: INtegral Cognitive REMediation; IQ: Intelligence Quotient; MDD: Major Depressive Disorder; PDQ: Perceived Deficit Questionnaire; RDQ: Remission from Depression Questionnaire; ROCF: Rey-Osterrieth Complex Figure; SCIP: Screening for Cognitive Impairment in Psychiatry; SF36-V2: 36-Item Short Form Health Survey, Version 2; STROOP: Stroop Color and Word Test; TAU: Treatment As Usual; TMT-A: Trail Making Test Part A; TMT-B: Trail Making Test Part B; TOL: Tower of London; WAIS-IV: Wechsler Adult Intelligence Scale version-IV; WCST: Wisconsin Card Sorting Test

\section{Acknowledgements}

We thank Dr. Ignasi Gich, from the Department of Epidemiology (Hospital de la Santa Creu i Sant Pau, Barcelona), for his help in the randomization process.

\section{Funding}

This study is financed by a governmental grant from the Spanish Ministry of Science, Innovation and Universities, Instituto Carlos III. Project number: PI17/00056. Duration: from 1th January 2018 to 31th December 2020. This funding source had no role in the design of this study and will not have any role during its execution, analyses, interpretation of the data, or decision to submit results.

\section{Availability of data and materials}

This is an ongoing study and the investigators are currently collecting data. Therefore, any publication containing the results of this study has not been published.

All the investigators collaborating in this study will have access to the final trial dataset. All writings and presentations will be performed by the members of the research team following the standard guidelines of dissemination policy.

\section{Authors' contributions}

MVG drafted the manuscript which was critically reviewed by MJP. MJP designed the study with the collaboration of BR, EMM, AMA, MSB and NC. $\mathrm{SGS}, \mathrm{CMB}$, JT, JPB, JDA, DP were involved in the set-up of the study, providing information upon the availability of facilities and clinical settings. MVG, EMM, AMA, CMB, MSB and MJP reviewed the theoretical rationale for manuscript preparation. JT, JDA, DP and MJP defined the study design and the statistical approach for the manuscript. BR, EMM, SGS, AMA, CMB, JT, JPB, $J D A, D P, M S B, N C$ read and approved the final version manuscript.

\section{Ethics approval and consent to participate}

This study is approved by the Ethical Committee for Clinical Research (in Spanish, Comité Ético de Investigación Clínica) from the Hospital de la Santa Creu i Sant Pau, project number IIBSP-RID-2017-107. Protocol version 1, 14th February 2018.

Central Clinical Research and Clinical Trials Unit (CCRCTU), Research Institute of the Hospital de Sant Pau, will monitor through periodic visits the correct progression of the project, alerting from protocol deviations. They will be constantly aware of every single step of the project.

All participants will receive extended information about the study and must give their written informed consent prior to participate in the study. This study does not pose any risk to the participants.

\section{Consent for publication}

Written informed consent from participants will be obtained prior to the inclusion to the study.

\section{Competing interests}

MJP has received honoraria as academic from Lundbeck. JDA has received consulting and/or lecture honoraria from Lundbeck, Pfizer and Qualigen. NC declares that he has received honoraria as consultant, advisor or CME speaker from Janssen, Lundbeck, Pfizer, Exeltis and MSD. The rest of the authors declare that they have no competing interests.

\section{Publisher's Note}

Springer Nature remains neutral with regard to jurisdictional claims in published maps and institutional affiliations.

\section{Author details}

'Department of Psychiatry, Hospital de la Santa Creu i Sant Pau, Centro de Investigación Biomédica en Red de Salud Mental (CIBERSAM), Institut d'Investigació Biomèdica Sant Pau (IBB-Sant Pau), Universitat Autònoma de Barcelona (UAB), Sant Antoni Ma Claret 167, 08025 Barcelona, Catalonia, Spain. ${ }^{2}$ Bipolar and Depressive Disorders Unit, Institute of Neurosciences, Hospital Clínic de Barcelona, Centro de Investigación Biomédica en Red de Salud Mental (CIBERSAM), IDIBAPS, University of Barcelona (UB), Barcelona, Catalonia, Spain. ${ }^{3}$ Mental Health Unit, Hospital Universitari Parc Taulí, Centro de Investigación Biomédica en Red de Salud Mental (CIBERSAM), Institut d'Investigació i Innovació Parc Taulí (I3PT), Universitat Autònoma de Barcelona (UAB), Sabadell, Barcelona, Catalonia, Spain.

Received: 7 February 2019 Accepted: 12 April 2019

Published online: 06 May 2019

\section{References}

1. Mclntyre RS, Cha DS, Soczynska JK, Woldeyohannes HO, Gallaugher LA, Kudlow $P$, et al. Cognitive deficits and functional outcomes in major depressive disorder: determinants, substrates, and treatment interventions. Depress Anxiety. 2013;30:515-27.

2. Chakrabarty T, Hadjipavlou G, Lam RW. Cognitive dysfunction in major depressive disorder: assessment, impact, and management. J Lifelong Learn Psychiatry. 2016;14:194-206.

3. Jaeger J, Berns S, Uzelac S, Davis-Conway S. Neurocognitive deficits and disability in major depressive disorder. Psychiatry Res. 2006;145:39-48.

4. Buist-Bouwman MA, Ormel J, de Graaf R, de Jonge P, van Sonderen E, Alonso J, et al. Mediators of the association between depression and role functioning. Acta Psychiatr Scand. 2009;118:451-8.

5. Baune BT, Miller R, McAfoose J, Johnson M, Quirk F, Mitchell D. The role of cognitive impairment in general functioning in major depression. Psychiatry Res. 2010;176:183-9.

6. Salagre E, Solé B, Tomioka Y, Fernandes BS, Hidalgo-Mazzei D, Garriga M, et al. Treatment of neurocognitive symptoms in unipolar depression: a systematic review and future perspectives. J Affect Disord. 2017;221:205-21.

7. Greer TL, Sunderajan P, Grannemann BD, Kurian BT, Trivedi MH. Does duloxetine improve cognitive function independently of its antidepressant effect in patients with major depressive disorder and subjective reports of cognitive dysfunction? Depress Res Treat. 2014:1-13.

8. Al-Sukhni M, Maruschak N, Mcintyre RS. Vortioxetine: a review of efficay, safety and tolerability with a focus on cognitive symptoms in major depressive disorder. Expert Opin Drug Saf. 2016;14(8):1291-304.

9. Frampton JE. Vortioxetine: a review in cognitive dysfunction in depression. Drugs. 2016. https://doi.org/10.1007/s40265-016-0655-3.

10. Wykes T, Huddy V, Cellard C, Mcgurk SR, Czobor P. A meta-analysis of cognitive remediation for schizophrenia: methodology and effect sizes. Am J Psychiatry. 2011;168:472-85.

11. Penadés R, Catalán R, Pujol N, Masana G, García-Rizo C, Bernardo M. The integration of cognitive remediation therapy into the whole psychosocial rehabilitation process: an evidence-based and person-centered approach. Rehabil Res Pract. 2012:1-8.

12. Elgamal S, McKinnon MC, Ramakrishnan K, Joffe RT, MacQueen GM. Successful computer-assisted cognitive remediation therapy in patients with unipolar depression : a proof of principle study. Psychol Med. 2007;37:1229-38.

13. Naismith SL, Redoblado-Hodge MA, Lewis SJG, Scott EM, Hickie IB. Cognitive training in affective disorders improves memory: a preliminary study using the NEAR approach. J Affect Disord. 2010;121:258-62.

14. Bowie CR, Gupta M, Holshausen K, Jokic R, Best M, Milev R. Cognitive remediation for treatment-resistant depression effects on cognition and functioning and the role of online homework. J Nerv Ment Dis. 2013;201:680-5.

15. Trapp W, Engel S, Goeran H, Lautenbacher S, Gallhofer B. Cognitive remediation for depressed inpatients: results of a pilot randomized controlled trial. Aust New Zeal J Psychiatry. 2016;50:46-55.

16. Conradi HJ, Ormel J, de Jonge P. Presence of individual (residual) symptoms during depressive episodes and periods of remission: a 3-year prospective study. Psychol Med. 2011;41:1165-74. 
17. Vicent-Gil M, Keymer-Gausset A, Serra-Blasco M, Carceller-Sindreu M, de Diego-Adeliño J, Trujols J, et al. Cognitive predictors of illness course at 12 months after first-episode of depression. Eur Neuropsychopharmacol. 2018;28:529-37.

18. Cicerone KD, Langenbahn DM, Braden C, Malec JF, Kalmar K, Fraas M, et al. Evidence-based cognitive rehabilitation: updated review of the literature from 2003 through 2008. Arch Phys Med Rehabil. 2011;92:519-30.

19. Choi J, Twamley EW. Cognitive rehabilitation therapies for Alzheimer's disease: a review of methods to improve treatment engagement and selfefficacy. Neuropsychol Rev. 2013;23:48-62.

20. Motter JN, Pimontel MA, Rindskopf D, Devanand DP, Doraiswamy PM, Sneed JR. Computerized cognitive training and functional recovery in major depressive disorder: a meta-analysis. J Affect Disord. 2016;189:184-91.

21. Martínez-Arán A, Torrent C, Solé B, Bonnín CM, Rosa AR, Sánchez-Moreno JVE. Functional remediation for bipolar disorder. Clin Pract Epidemiol Ment Heal. 2011;7:112-6.

22. Torrent C, Del Mar Bonnin C, Martínez-Arán A, Valle J, Amann BL, GonzálezPinto A, et al. Efficacy of functional remediation in bipolar disorder: a multicenter randomized controlled study. Am J Psychiatry. 2013;170:852-9.

23. Solé B, Bonnin CM, Mayoral M, Amann BL, Torres I, González-Pinto A, et al. Functional remediation for patients with bipolar II disorder: improvement of functioning and subsyndromal symptoms. Eur Neuropsychopharmacol. 2015;25:257-64

24. Bonnin CM, Torrent C, Arango C, Amann BL, Solé B, González-Pinto A, et al. Functional remediation in bipolar disorder: 1-year follow-up of neurocognitive and functional outcome. Br J Psychiatry. 2016;208:87-93.

25. Pino O, Guilera G, Rojo JE, Gómez-Benito J, Bernardo M, Crespo-Facorro B, et al. Spanish version of the screen for cognitive impairment in psychiatry (SCIP-S): psychometric properties of a brief scale for cognitive evaluation in schizophrenia. Schizophr Res. 2008;99:139-48.

26. Guilera G, Pino O, Gómez-Benito J, Rojo JE, Vieta E, Tabarés-Seisdedos R, Segarra N, Martínez- Arán A, Franco M, Cuesta MJ, Crespo-Facorro B, Bernardo M, Purdon SE, Díez T, Rejas J. Clinical usefulness of the screen for cognitive impairment in psychiatry (SCIP-S) scale in patients with type I bipolar disorder. Health Qual Life Outcomes. 2009:7-28.

27. Bonnín CM, Martínez-Arán A, Reinares M, Valentí M, Solé B, Jiménez E, et al. Thresholds for severity, remission and recovery using the functioning assessment short test (FAST) in bipolar disorder. J Affect Disord. 2018;240:57-62

28. Rosa AR, Sánchez-Moreno J, Martínez-Aran A, Salamero M, Torrent C, Reinares $M$, et al. Validity and reliability of the functioning assessment short test (FAST) in bipolar disorder. Clin Pract Epidemiol Ment Heal. 2007;3:1-10.

29. Strober LB, Binder A, Nikelshpur OM, Chiaravalloti N, DeLuca J. The perceived deficits questionnaire: perception, deficit, or distress? Int J MS Care. 2016;18:183-90.

30. Lam RW, Saragoussi D, Danchenko N, Rive B, Lamy FX, Brevig T. Psychometric validation of perceived deficits questionnaire - depression (PDQ-D) in patients with major depressive disorder (MDD). Value Heal. 2013; 16:A330-QL4.

31. Hamilton M. A rating scale for depression. J Neurol Neurosurg Psychiatry. 1960;23:56-62.

32. Bobes J, Bulbena A, Luque A, Dal-Ré R, Ballesteros J, Ibarra N. Evaluación psicométrica comparativa de las versiones en español de 6, 17 y 21 ítems de la escala de valoración de Hamilton para la evaluación de la depresión. Med Clin. 2003:120:693-700.

33. Zimmerman M, Martinez JH, Attiullah N, Friedman M, Toba C, Boerescu DA, et al. A new type of scale for determining remission from depression: the remission from depression questionnaire. J Psychiatr Res. 2013;47:78-82.

34. Ware JE. SF-36 health survey update. Spine (Phila Pa 1976). 2000;25:3130-9.

35. Wechsler D. Weschler Adult Intelligence Scale (WAIS-IV). 4th ed. San Antonio, Texas: Pearson; 2008.

36. Conners CK. Conners continuous performance test (CPT-3). NY: Towanda; 2014.

37. Delis DC, Kramer JH, Kaplan E, Ober BA. California verbal learning test: adult version. San Antonio, Texas; 1987

38. Rey A. L'examen clinique en psychologie. Paris: Presses Universitaires de France; 1941.

39. Tombaugh TN. Trail making test a and B: normative data stratified by age and education. Arch Clin Neuropsychol. 2004;19:203-14.

40. Golden CJ. Stroop colour and word test. Chicago: Stoelting; 1978.
41. Heaton RK. Wisconsin card sorting test manual. Odessa: psychological assessment. Resources. 1981.

42. Portella MJ, Marcos-Bars T, Rami-González L, Navarro-Odriozola V, GastóFerrer C, Salamero M. Torre de Londres: planificación mental, validez y efecto techo. Rev Neurol. 2003:37:210-3.

43. Peña-Casanova J, Quiñones-Úbeda S, Gramunt-Fombuena N, QuintanaAparicio M, Aguilar M, Badenes D, et al. Spanish multicenter normative studies (NEURONORMA project): norms for verbal fluency tests. Arch Clin Neuropsychol. 2009;24:395-411.

44. Casals-Coll M, Sánchez-Benavides G, Quintana M, Manero RM, Rognoni T, Calvo $L$, et al. Estudios normativos españoles en población adulta joven (proyecto NEURONORMA jóvenes): Normas para los test de fluencia verbal. Neurologia. 2013;28:33-40.

45. Vieta E, Torrent C, Martínez-Arán A. Functional remediation for bipolar disorder; 2014

46. Lewinsohn PM, Antonuccio DO, Steinmetz JL, Teri L. The coping with depression course: a psychoeducational intervention for unipolar depression. Eugene. Oregon: Castalia Publishing; 1984.

47. Casañas R, Catalán R, del Val JL, Real J, Valero S, Casas M. Effectiveness of a psycho-educational group program for major depression in primary care: a randomized controlled trial. BMC Psychiatry. 2012;12:230.

48. Aragonès E, Cardoner N, Colom F, Lopez-Cortacans G. Guía de Buena Práctica Clínica: Psicoeducación en pacientes con Depresión: Organización Médica Colegial; 2013.

49. Sobocki P, Jönsson B, Angst JRC. Cost of depression in Europe. J Ment Heal Policy Econ. 2006:9:87-98.

\section{Ready to submit your research? Choose BMC and benefit from:}

- fast, convenient online submission

- thorough peer review by experienced researchers in your field

- rapid publication on acceptance

- support for research data, including large and complex data types

- gold Open Access which fosters wider collaboration and increased citations

- maximum visibility for your research: over $100 \mathrm{M}$ website views per year

At $\mathrm{BMC}$, research is always in progress.

Learn more biomedcentral.com/submissions 